\title{
A GENERALISATION OF DESCARTES' RULE OF SIGNS
}

\author{
DANIEL V. TOKAREV
}

(Received 18 August 2010; accepted 25 May 2011)

Communicated by A. M. Hassell

\begin{abstract}
A generalisation of Descartes' rule of signs to other functions is derived and a bound for the number of positive zeros of a class of integral transforms is deduced from that. A more precise rule of signs is also discussed in the light of these results.
\end{abstract}

2010 Mathematics subject classification: primary 26C10.

Keywords and phrases: Descartes' rule of signs, zeros of polynomials, zeros of integral transforms.

\section{Introduction and main results}

The classical rule of signs due to Descartes provides an elementary upper bound for the number of positive zeros of a polynomial, namely, the number of sign changes of its coefficients. Since its publication in Descartes' monumental La Géométrie in 1637, there has been a substantial body of research on the rule (see, for example, [1, 5$8,10])$. While these papers have focused on presenting simple proofs of the rule, investigating whether the bound is tight, constructing polynomials for which it is exact, and extending the rule to higher dimensions, this note generalises Descartes' rule of signs (DRS) to other functions and derives a bound on the number of zeros of a class of integral transforms in terms of the number of crossings of the integrator function. This also enables us to understand a more natural formulation of the rule, which is illustrated with a simple extension of the classical result.

The classical rule of signs states that, for a sequence of real coefficients $\left\{a_{i}\right\}_{i=0}^{n}$ and a sequence of strictly increasing positive integer powers $\left\{b_{i}\right\}_{i=0}^{n}$, the polynomial $p(x)=\sum_{i=0} a_{i} x^{b_{i}}$ has no more positive real zeros than the number of sign changes of $\left\{a_{i}\right\}_{i=0}^{n}$. In the rest of this paper, zeros of a function mean positive real zeros; and all functions and sequences are real valued.

More generally, we say that a sequence of functions $\left\{u_{j}(s)\right\}$ satisfies DRS if, for any sequence $\left\{b_{j}\right\}_{j=0}^{n}$, the number of positive zeros of $\sum_{j=0}^{n} b_{j} u_{j}(s)$ is no greater than 
the number of sign changes of $\left\{b_{j}\right\}$. We also say that a function $\phi(s, t)$ defined on $\mathbb{R}_{+}^{2}$ satisfies DRS if the sequence of functions (of $\left.s\right)\left\{\phi\left(s, t_{j}\right)\right\}_{j=0}^{m}$ satisfies DRS for any choice of the $t_{j}$ such that $0<t_{1}<t_{2}<\cdots<t_{m}$. Examples of such functions include $\phi(s, t)=s^{t}$ and $\phi(s, t)=e^{-s t}$ (see, for example $\left.[2,10]\right)$.

We will prove the following result.

THEOREM 1. Let $\left\{\mu_{j}\right\}$ be a sequence of positive measures on disjoint subsets of $\mathbb{R}^{+}$such that the support of the measure $\mu_{j}$ is to the left of the support of the measure $\mu_{k}$ if $j<k$. Suppose that the functions $\phi(s, t)$ satisfy DRS and are $\mu_{j}$-integrable on $\mathbb{R}^{+}$(as functions of $t$ ). Define

$$
u_{j}(s):=\int_{0}^{\infty} \phi(s, t) \mu_{j}(d t) .
$$

Then the sequence $\left\{u_{j}(s)\right\}$ satisfies DRS.

Remark 2. The classical rule of signs is the special case when $\phi(s, t)=s^{t}$ and the measure $\mu$ is supported on the integers. Indeed,

$$
p(x)=\sum_{i=0}^{n} a_{i} x^{i}=\log (x) \int_{0}^{\infty} x^{t} \mu(d t),
$$

where $\mu$ is generated as follows: if $(\alpha, \beta] \subseteq(j, j+1]$, where $j=0,1,2, \ldots$, then

$$
\mu((\alpha, \beta])=-(\beta-\alpha) \sum_{i=0}^{j} a_{i} .
$$

It would be interesting to see whether a characterisation of the class of sequences that satisfy DRS may be found in this manner.

Before we proceed to our main result, we recall some standard results and give some definitions, which will be familiar to many readers.

Theorem 3 (Hahn decomposition theorem). Given a measurable space $(X, \Sigma)$ and a signed measure $\mu$ on the $\sigma$-algebra $\Sigma$, there exist sets $S^{+}$and $S^{-}$in $\Sigma$ with the following properties.

(1) $S^{+} \cup S^{-}=X$ and $S^{+} \cap S^{-}=\emptyset$.

(2) $\mu(E) \geq 0$ for each $E \in \Sigma$ such that $E \subseteq S^{+}$; that is, $S^{+}$is a positive set for $\mu$.

(3) $\mu(E) \leq 0$ for each $E \in \Sigma$ such that $E \subseteq S^{-}$; that is, $S^{-}$is a negative set for $\mu$.

This decomposition is essentially unique, in the sense that for any other pair $\left(T^{+}, T^{-}\right)$of measurable sets fulfilling the above three conditions, the symmetric differences $S^{+} \Delta T^{+}$and $S^{-} \Delta T^{-}$are $\mu$-null sets in the strong sense that every measurable subset of each of them has zero measure. The next result is a consequence.

Corollary 4 (Hahn-Jordan decomposition). Each signed measure $\mu$ may be written as a difference of positive measures $\mu^{+}$and $\mu^{-}$, at least one of which is finite. These measures may be defined by $\mu^{+}(E):=\mu\left(E \cap S^{+}\right)$and $\mu^{-}(E):=-\mu\left(E \cap S^{-}\right)$.

Given a signed measure $\mu$, we now define $v$, the number of crossings of $\mu$. 
Definition 5. When $S^{+}$has infinitely many connected components, we set $v=\infty$. Otherwise, $S^{+}$and $S^{-}$have only finitely many connected components $S_{1}^{+}, \ldots, S_{v_{+}}^{+}$ and $S_{1}^{-}, \ldots, S_{\nu_{-}}^{-}$. If $0 \in S_{1}^{+}$, then $0 \leq v_{+}-v_{-} \leq 1$ and $x_{1}^{+}<x_{1}^{-}<x_{2}^{+}<x_{2}^{-}<\cdots$ for any choice of $x_{i}^{ \pm} \in S_{i}^{ \pm}$. Let $v=v^{-}+v^{+}-1$ and define sets $Q_{1}, \ldots, Q_{v}$ by $Q_{1}:=S_{1}^{+}$, $Q_{2}:=S_{1}^{-}, Q_{3}:=S_{2}^{+}, Q_{4}:=S_{2}^{-}$, and so on. If $0 \in S_{1}^{-}$, then we reverse the roles of + and - .

In the special case where $\mu$ has a continuous density $F$, that is,

$$
\mu([a, b])=\int_{a}^{b} F(t) d t,
$$

$v$ is precisely the number of crossings of the positive $x$-axis by the graph of $F$.

Definition 6. The Riemann-Stieltjes integral of a real-valued function $f$ of a real variable with respect to a measure $\mu$, denoted by

$$
\int_{a}^{b} f(t) \mu(d t)
$$

is defined to be the limit (if it exists), as $\max \left\{\mid x_{i}-x_{i-1}\right\}$ approaches zero, of the approximating sums

$$
S(f, \mu)=\sum_{i=1}^{n} f\left(c_{i}\right) \mu\left(\left(x_{i-1}, x_{i}\right]\right),
$$

where $a=x_{0}<x_{1}<\cdots<x_{n}=b$ and $c_{i} \in\left[x_{i-1}, x_{i}\right]$.

Now we can state our main result.

Theorem 7. Let $\phi(s, t)$ satisfy Descartes' rule of signs, let $\mu$ be a signed measure on $\mathbb{R}^{+}$, and let

$$
\Phi(s):=\int_{0}^{\infty} \phi(s, t) \mu(d t) .
$$

Then the number of positive zeros of $\Phi$ does not exceed $\nu$, the number of crossings of $\mu$.

REMARK 8 . This can be easily applied to the example of the Laplace transform, since $\phi(s, t)=e^{-t s}$ satisfies DRS. When the signed measure $\mu$ admits a continuous density, the bound can be given by the number of zeros of the density, but this bound may be less tight, since the density may have zeros on an interval where the measure does not change sign.

The preceding result formulates the rule of signs in terms of the number of sign changes in the Hahn-Jordan decomposition of the signed measure $\mu$. This suggests another rule of signs, which turns out to be more precise than the classical rule.

TheOREM 9. Let $p(x)=\sum_{i=0}^{n} a_{i} x^{i}$ and $p_{j}(x)=\sum_{i=0}^{j} a_{i} x^{i}$. Then the number of zeros of $p(x)$ in the interval $(0, m)$ is no greater than the number of sign changes of $\left\{p_{j}(m)\right\}_{j=0}^{n}$. 
REMARK 10. This rule is sensitive, not only to the alternating signs of the coefficients, but also to their magnitudes. If $m$ is greater than the largest zero of $p(x)$, then we have a bound on the number of positive roots of $p$. Descartes' rule of signs follows as an immediate corollary, since a change of sign between $p_{j}(m)$ and $p_{j+1}(m)$ is only possible if there is a change of sign between $a_{j}$ and $a_{j+1}$, but of course this is not sufficient in general.

Cauchy [3] gave a simple upper bound for the largest zero (in absolute value), namely, $1+\max \left\{\left|a_{i} / a_{n}\right|: 1 \leq i \leq n-1\right\}$. We can set $m$ equal to this value and find a bound on the total number of zeros. For instance, if $p(x)=10-x+x^{2}-2 x^{3}$, then there are three changes of sign and thus there are at most three zeros, according to DRS. The Cauchy upper bound for the largest zero is 6 . Finding the values $\left\{p_{j}(6)\right\}$, we obtain $\{10,4,40,-398\}$; there is one sign change. And, in fact, there is precisely one positive zero, at approximately 1.78659 .

\section{Proofs}

In order to prove our results, we employ the generalisation of DRS due to Carnicer and Peña [2] which involves sign regular sequences. We adopt the method of [9].

A sequence of functions $\left\{u_{1}, \ldots, u_{n}\right\}$ defined on an interval $I$ is said to be strictly sign regular if, for any choice of points $s_{1}, s_{2}, \ldots, s_{m}$ in $I$ such that $s_{1}<s_{2}<\cdots<s_{m}$, all minors of the matrix $\left(u_{j}\left(s_{i}\right)\right)_{i \leq m, j \leq n}$ have the same strict sign. For instance, sequences of the form $\left\{x^{t_{1}}, \ldots, x^{t_{n}}\right\}$ and $\left\{e^{-t_{1} x}, \ldots, e^{-t_{n} x}\right\}$, where $0<t_{1}<t_{2}<\cdots<t_{n}$, are strictly sign regular on $\mathbb{R}^{+}$; see, for example, [2, p. 34]. By [2, Proposition 2.6], a system of functions $\left\{u_{1}, \ldots, u_{n}\right\}$ satisfies DRS if and only if it is strictly sign regular.

We are now ready to prove Theorem 1.

Proof of Theorem 1. First, since $\phi(s, t)$ satisfies DRS, the $\operatorname{sign}$ of $\operatorname{det}\left(\phi\left(s_{i}, t_{j}\right)\right)_{i, j \leq k}$ when $k \leq v$ is the same for any choice of $s_{i}$ and $t_{j}$ such that $0<s_{1}<\cdots<s_{k}$ and $0<t_{1}<\cdots<t_{k}$.

Observe that a minor of $\left(u_{j}\left(s_{i}\right)\right)_{j \leq v, i \leq m}$ admits an integral representation in terms of the corresponding minor of $\left(\phi\left(s_{j}, t_{i}\right)\right)_{j \leq v, i \leq m}$. Indeed, without loss of generality, consider the determinant of $\left(u_{j}\left(s_{i}\right)\right)_{i, j \leq k}$, where $0<s_{1}<\cdots<s_{k}$. By Fubini's theorem,

$$
\begin{aligned}
\sum_{\sigma} \operatorname{sgn}(\sigma) \prod_{j=1}^{k} u_{j}\left(s_{\sigma(j)}\right) & =\sum_{\sigma} \operatorname{sgn}(\sigma) \prod_{j=1}^{k} \int_{0}^{\infty} \phi\left(s_{\sigma(j)}, t\right) \mu_{j}(d t) \\
& =\int \cdots \int \sum_{\sigma} \operatorname{sgn}(\sigma)\left(\prod_{j=1}^{k} \phi\left(s_{\sigma(j)}, t_{j}\right)\right) \mu_{1}(d t) \cdots \mu_{k}(d t) \\
& =\int \cdots \int \operatorname{det}\left(\phi\left(s_{\sigma(j)}, t_{j}\right)\right) \mu_{1}(d t) \cdots \mu_{k}(d t),
\end{aligned}
$$

where $\mu_{j}(A):=\mu\left(A \cap Q_{j}\right)$ and $\operatorname{sgn}(\sigma)$ is the signature of the permutation $\sigma$ of the set $\{1, \ldots, k\}$. Note that $t_{0}<t_{1}<\cdots<t_{k}$ by construction in the integrand in the last displayed formula, because $t_{j} \in Q_{j}$; and, since $\phi(s, t)$ satisfies DRS, the minor of 
$\left(u_{j}\left(s_{i}\right)\right)_{j \leq v, i \leq m}$ is strictly sign regular: thus, the determinant is positive and so is the iterated integral. Hence, the sequence $\left\{\int_{[0, \infty)} \phi(s, t) \mu_{j}(d t)\right\}$ is strictly sign regular and therefore satisfies DRS.

Proof of Theorem 7. We may rewrite our integral as

$$
\int_{0}^{\infty} \phi(s, t) \mu(d t)=\sum_{j=1}^{v} \int_{Q_{j}} \phi(s, t) \mu(d t)=\sum_{j=1}^{v}(-1)^{j+1} \int_{0}^{\infty} \phi(s, t)\left|\mu_{j}(d t)\right|,
$$

where $\left|\mu_{j}(A)\right|:=\left|\mu\left(A \cap Q_{j}\right)\right|$ for a set $A$. Since $\phi(s, t)$ satisfies DRS and $\left\{\mu_{j}\right\}$ is a sequence of positive measures on disjoint subsets of $\mathbb{R}^{+}$by assumption, Theorem 1 applies and the required result follows.

Proof of Theorem 9. Let $x:=m s$ in (1). When $m>0$ and $0<x<1$,

$$
p(m s)=\sum_{i=0}^{n} a_{i} s^{i}=\log (s) \int_{0}^{\infty} s^{t} \mu(d t)
$$

where $\mu$ is a measure supported on the positive integers and generated as follows: if $(\alpha, \beta] \subseteq(j, j+1]$, where $j=0,1,2, \ldots$, then

$$
\mu((\alpha, \beta])=-(\beta-\alpha) \sum_{i=0}^{j} a_{i} .
$$

The number of zeros of $p(x)$ in $(0, m)$ is the same as the number of zeros of $p(m s)$ in $(0,1)$, which cannot be greater than the number of crossings of $\mu$, by Theorem 7 . This is of course exactly the number of sign changes of $\left\{\sum_{i=0}^{j} a_{i} m^{j}\right\}=\left\{p_{j}(m)\right\}$.

REMARK 11. Interestingly, Theorem 9 may be proven trivially from the classical rule of signs by writing

$$
q(x):=\frac{p(x)}{1-x}=: \sum_{j=0}^{\infty} b_{j} x^{j}
$$

recognising that $b_{j}=\sum_{i=0}^{j} a_{i}$, and noting that $p(x)$ and $q(x)$ have the same number of zeros, while $q(x)$ has no more zeros than the number of sign changes of $b_{j}$. Here we have assumed a well-known extension of DRS to power series (see [4]), since $q(x)$ is an infinite power series unless $p(1)=0$. We noted in Remark 10 that this rule implies the classical rule of signs and here we see that it follows from its version for power series.

\section{Acknowledgement}

Our thanks go to an anonymous referee for pointing out Remark 11.

\section{References}

[1] B. Anderson, J. Jackson and M. Sitharam, 'Descartes Rule of Signs revisited', Amer. Math. Monthly 105 (1998), 447-451.

[2] J. Carnicer and J. M. Peña, 'Characterizations of the optimal Descartes rules of signs', Math. Nachr. 189 (1998), 33-48. 
[3] A. L. Cauchy, 'Exercises de mathématiques. IV Année'. Chez de Bure Frères, Paris, 1829.

[4] D. R. Curtis, 'Recent extensions of Descartes' rule of signs', Ann. Math. 19 (1918), 251-278.

[5] D. J. Grabine, 'Descartes' Rule of Signs: another construction', Amer. Math. Monthly 106 (1999), 854-855.

[6] U. Itenberg and M.-F. Roy, 'Multivariate Descartes' Rule', Beiträge Algebra Geom. 37 (1996), $337-346$.

[7] V. Komornik, 'Another short proof of Descartes's Rule of Signs', Amer. Math. Monthly 113 (2006), 829-830.

[8] M. Schmitt, 'New designs for the Descartes Rule of Signs', Amer. Math. Monthly 111 (2004), 159-164.

[9] D. Tokarev and K. Borovkov, 'On the expectations of maxima of sets of independent random variables', Statist. Probab. Lett. 79 (2009), 2381-2388.

[10] X. Wang, 'A simple proof of Descartes's Rule of Signs', Amer. Math. Monthly 111 (2004), $525-526$.

DANIEL V. TOKAREV, School of Mathematical Sciences, Monash University, Clayton 3800, Australia

e-mail: daniel.tokarev@monash.edu 